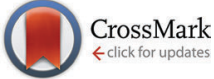

Cite this: Phys. Chem. Chem. Phys., 2017, 19, 2683

Received 25th November 2016 Accepted 21st December 2016

DOI: $10.1039 / \mathrm{c} 6 \mathrm{cp} 08070 \mathrm{~g}$

www.rsc.org/pccp

\section{Focused electron beam based direct-write fabrication of graphene and amorphous carbon from oxo-functionalized graphene on silicon dioxide $\dagger$}

\author{
Severin Schindler, $\ddagger^{d}$ Florian Vollnhals, $\neq \S^{c}$ Christian E. Halbig, ${ }^{b}$ Hubertus Marbach, ${ }^{c}$ \\ Hans-Peter Steinrück, ${ }^{C}$ Christian Papp* ${ }^{\star c}$ and Siegfried Eigler*abd
}

\begin{abstract}
Controlled patterning of graphene is an important task towards device fabrication and thus is the focus of current research activities. Graphene oxide (GO) is a solution-processible precursor of graphene. It can be patterned by thermal processing. However, thermal processing of GO leads to decomposition and $\mathrm{CO}_{2}$ formation. Alternatively, focused electron beam induced processing (FEBIP) techniques can be used to pattern graphene with high spatial resolution. Based on this approach, we explore FEBIP of $\mathrm{GO}$ deposited on $\mathrm{SiO}_{2}$. Using oxo-functionalized graphene (oxo-G) with an in-plane lattice defect density of $1 \%$ we are able to image the electron beam-induced effects by scanning Raman microscopy for the first time. Depending on electron energy (2-30 keV) and doses (50-800 mC m$~^{-2}$ ) either reduction of GO or formation of permanent lattice defects occurs. This result reflects a step towards controlled FEBIP processing of oxo-G.
\end{abstract}

\section{Introduction}

Graphene exhibits outstanding electronic properties and potential applications ranging from electronic devices over heat spreaders and membranes to sensing applications. ${ }^{1-3}$ Graphene oxide (GO) is an oxidized derivative of graphene prepared from graphite and the properties depend on the preparation method and type of graphite: ${ }^{4-6}$ it can be processed from aqueous dispersions and

\footnotetext{
${ }^{a}$ Freie Universität Berlin, Institute of Chemistry and Pharmacy, Takustraße 3, 14195 Berlin, Germany. E-mail: Siegfried.eigler@fu-berlin.de; Tel: $+49(0) 3083861851$

${ }^{b}$ Department of Chemistry and Pharmacy and Institute of Advanced Materials and Processes (ZMP), Friedrich-Alexander-Universität Erlangen-Nürnberg (FAU), Dr.-Mack Str. 81, 90762 Fürth, Germany

${ }^{c}$ Department of Chemistry and Pharmacy, Friedrich-Alexander-Universität

Erlangen-Nürnberg (FAU), Egerlandstraße 1, 91058 Erlangen, Germany. E-mail: christian.papp@fau.de

${ }^{d}$ Department of Chemistry and Chemical Engineering,

Chalmers University of Technology, Kemivägen 10, 41258 Göteborg, Sweden

$\dagger$ Electronic supplementary information (ESI) available. See DOI: 10.1039/c6cp08070g

$\$$ S. Schindler and F. Vollnhals contributed equally to this work.

$\S$ Present address: Advanced Instrumentation for Ion Nano-Analytics (AINA), Luxembourg Institute of Science and Technology (LIST), 41 rue du Brill, L-4422 Belvaux, Luxembourg.
}

converted to conductive carbon. ${ }^{7}$ However, the carbon lattice bears manifold defects due to the harsh oxidative preparation protocols. ${ }^{8}$ Nevertheless, various reduction methods such as hydrated electrons, plasmas, ${ }^{9,10}$ ultraviolet light, ${ }^{11}$ or chemical reduction agents were reported to prepare reduced GO, a carbon material with an irregular, but conductive carbon framework. ${ }^{7,12,13}$

Recently, scanning Raman microscopy (SRM) has been developed as the method of choice to visualize structures in graphene. ${ }^{14,15}$ SRM can image in-plane lattice defect densities up to an upper limit of $2-3 \% .^{16,17}$ For oxo-functionalized graphene (oxo-G), which can be prepared in high quality with defect densities as low as $0.05 \%$, SRM has been applied to visualize the density of defects. ${ }^{18-20}$ Recently, the intact carbon framework of this low defect material was imaged by highresolution transmission electron microscopy. ${ }^{21}$ Raman spectroscopy can also be used to evaluate the efficiency of the reduction of oxo-G with a relatively high defect density of about $1 \%$, which is also relevant for conventional GO.

Patterning of graphene on the $\mathrm{nm}$ to $\mu \mathrm{m}$ scale is of general interest for an economic and environmentally friendly preparation of device structures. ${ }^{22}$ In particular, thermal methods, such as a laser or a hot tip of an atomic force microscope, have been used to directly pattern "graphene". ${ }^{23-25}$ However, these thermal treatments of GO result in a loss of carbon from the graphene lattice via $\mathrm{CO}_{2}$ formation and other organic fragments. ${ }^{8,26,27}$ Thus, additional permanent lattice defects are introduced and therefore, more likely, amorphous conductive carbon is produced rather than undisturbed graphene. ${ }^{28,29} \mathrm{~A}$ possible post treatment at very high temperatures of above $1000{ }^{\circ} \mathrm{C}$ would be necessary to induce lattice rearrangement leading to graphene. ${ }^{30-32}$ As an alternative, also conductive atomic force microscopy was used to reduce $\mathrm{GO}$ at a specific potential. ${ }^{33}$

In this contribution, we follow a different approach for the patterning of graphene, namely focused electron beam induced processing (FEBIP) of oxo-G. FEBIP subsumes powerful techniques to lithographically pattern interfaces on the nanoscale, either by the dissociation of adsorbed precursor molecules ${ }^{34,35}$ or by the modification of the substrate itself, ${ }^{36}$ both by the impact of the 
A)
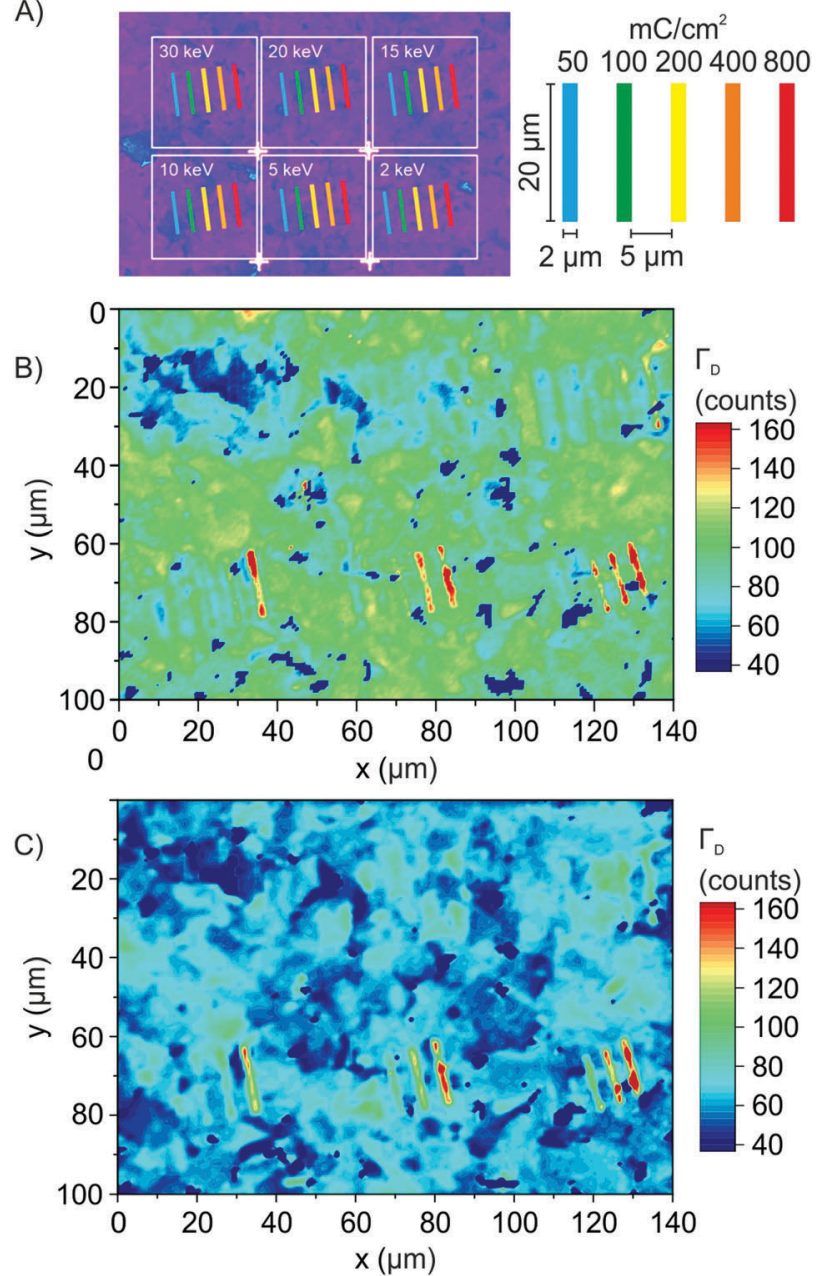

Fig. 1 (A) Scheme of the electron irradiation of oxo- $G$ flakes on a $\mathrm{SiO}_{2}$ $(300 \mathrm{~nm}) / \mathrm{Si}$ wafer. The color code indicates the dose ranging from 50 to $800 \mathrm{mC} \mathrm{cm}^{-2}$ for the beam energies between 2 and $30 \mathrm{keV}$. (B) Raman map of a film of oxo-G flakes mapping the full-width at half-maximum $\left(\Gamma_{\mathrm{D}}\right)$ of the Raman $\mathrm{D}$ peak after e-beam irradiation of different energy and doses. Red: broad $D$ peaks (low quality, amorphous), blue narrow $D$ peaks (better quality). (C) Raman map of the same area as in (B) of reduced oxo-G after FEBIP. Introduced areas of increased defect densities (red area) are permanent.

electron beam. Herein, we use the latter approach, that is, the direct impingement of an electron beam on oxo-G/SiO 2 (Fig. 1). When varying the beam energy and the electron beam exposure, we find either a reduction yielding intact graphene areas or an irreversible degradation of the carbon framework yielding amorphous carbon areas. This finding allows for the positive and negative patterning of graphene structures.

\section{Results}

Oxo-G was prepared based on our previously described method using potassium permanganate as an oxidant in sulfuric acid at an increased (around $25^{\circ} \mathrm{C}$ ) temperature during the purification procedure (see the ESI $\dagger$ for details and analysis, chemical sketch given in Fig. 2A). ${ }^{19,37} \mathrm{~A}$ film of oxo-G flakes was placed on a $\mathrm{Si} / 300 \mathrm{~nm} \mathrm{SiO}{ }_{2}$ substrate by a Langmuir-Blodgett technique.
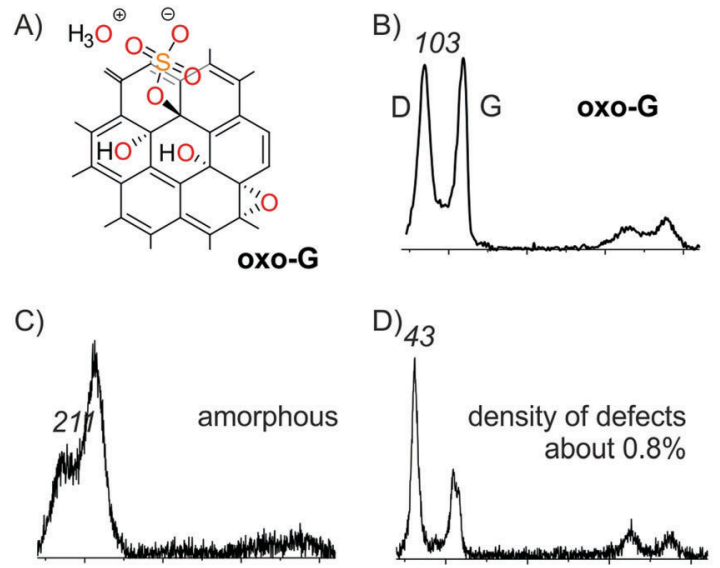

E)

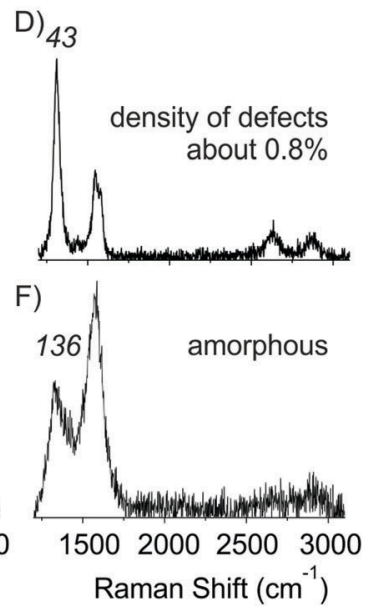

Fig. 2 (A) Chemical sketch of oxo-G. (B) Raman spectrum of oxo-G before treatment; $\Gamma_{\mathrm{D}}$ values are given in italic numbers. (C-F) Raman spectra of electron beam irradiated areas shown in Fig. $1 \mathrm{~B}$ at selected energies and doses. (C) $2 \mathrm{keV}$

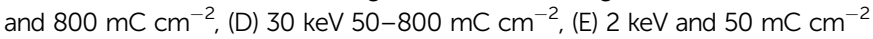
and (F) $10 \mathrm{keV}$ and $800 \mathrm{mC} \mathrm{cm}{ }^{-2} . \Gamma_{\mathrm{D}}$ values are given in italic numbers.

The density of lattice defects was determined to be about $1 \%$ by statistical Raman spectroscopy $\left(I_{\mathrm{D}} / I_{\mathrm{G}}=1.5 \pm 0.7 ; \Gamma_{2 \mathrm{D}}=66 \pm 19\right)$. Raman spectra show a broad D peak and G peak with a full-width at half-maximum $(\Gamma)>100 \mathrm{~cm}^{-1}$. These oxo-G films can be quantitatively reduced by HI/TFA, allowing for the determination of the lattice defect density by statistical Raman spectroscopy., ${ }^{9,15,38-41}$

The impact of an electron beam on these deposited oxo-G flakes was investigated using a standard scanning electron microscope (Zeiss Evo 40). All patterning steps were carried out using this microscope controlled by an Elphy Quantum pattern generator (Raith GmbH, Dortmund, Germany). The base pressure of the SEM is $2 \times 10^{-6} \mathrm{mbar}$. The step size during exposures was set to $9.6 \mathrm{~nm}$. The energy was varied between 2 and $30 \mathrm{keV}$, and doses of 50, 100, 200, 400 and $800 \mathrm{mC} \mathrm{cm}^{-2}$ were used to structure the oxo-G film on the $\mu \mathrm{m}$ scale (Fig. 1A). Subsequently, statistical Raman microscopy (SRM) was used to evaluate and visualize the impact of the electron beam on the quality of oxo-G or graphene. As a measure of quality we used $\Gamma_{\mathrm{D}}$ that narrows with the reduction, that is, with increasing quality of graphene. ${ }^{16,17}$ As evident from Fig. 1B, large variations of $\Gamma_{\mathrm{D}}$ are observed for different treatments. At $2 \mathrm{keV}$ (right lower corner) and high electron doses of 200, 400 and $800 \mathrm{mC} \mathrm{cm}^{-2}$, broad D peaks (red color) are observed - the absence of the 2D peak at $2700 \mathrm{~cm}^{-1}$ indicates that amorphous carbon was produced (Fig. 2C and F). ${ }^{42}$

Subsequent chemical reduction of the film by HI/TFA and SRM analysis (Fig. 1C) leaves the appearance unchanged, which indicates that the defect density in the radiated area is permanent. Thus, it is concluded that under the applied conditions lattice 
defects (in-plane) are introduced rather than functionalization defects (out-of-plane). On the other hand, at $30 \mathrm{keV}$ and doses

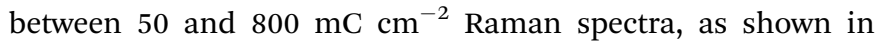
Fig. 2D, indicate the chemical reduction of oxo-G (Raman spectrum of oxo-G shown in Fig. 2B). The structure irradiated by the electron beam is, however, not visible in SRM (Fig. 1B, upper left corner). This is due to the fact that the patterned as well as the surrounding areas were completely reduced. This effect is also visible for regions irradiated at $20 \mathrm{keV}$. We propose that slow secondary electrons, generated by the interaction of primary $\left(\mathrm{SE}_{\mathrm{I}}\right)$ or backscattered electrons $\left(\mathrm{SE}_{\mathrm{II}}\right)$ with the $\mathrm{SiO}_{2}$ substrate, are responsible for this reduction. The lateral extension of the observed reduction indeed corresponds well with the range of emitted backscattered electrons (BSE), as determined from Monte-Carlo simulations performed with CASINO v2.42 (cf. Table S1, ESI $\dagger){ }^{43}$ Interestingly, the applied dose at $15 \mathrm{keV}$ plays a critical role, and we find that oxo-G is reduced at doses of 50 and $100 \mathrm{mC} \mathrm{cm}^{-2}$ and extended defects are introduced at 400 and $800 \mathrm{mC} \mathrm{cm}^{-2}$. This observation indicates that the electron beam can either produce more graphene-like material inducing defunctionalization or harm the carbon framework, introducing lattice defects, depending on the exposure conditions.

Temperature-dependent patterning experiments (from room temperature to $60{ }^{\circ} \mathrm{C}$ ), with $15 \mathrm{keV}$ and doses between 50 and $800 \mathrm{mC} \mathrm{cm}^{-2}$, suggest that the reduction is not a thermally activated process in this temperature range. Notably, we find no significant difference in the Raman spectra of an irradiated film of oxo-G flakes and reduced oxo-G, (SRM results depicted in Fig. S1A and $\mathrm{B}, \mathrm{ESI} \dagger \Gamma_{\mathrm{D}}$ peak maps), within the experimental error.

The structures that are produced will certainly depend on the size of the electron beam and the energy dependent BSE area. However, here we demonstrate that a structure patterned with a width of $2 \mu \mathrm{m}$ can be resolved in Raman microscopy images that we recorded with a scanning increment of $75 \mathrm{~nm}$, fairly below the maximum of optical resolution limit of roughly $335 \mathrm{~nm}$. We find that for higher beam energies the material is reduced (blue) in a large distance around the irradiated structures, and that the defective structures are confined to the patterned areas (red). Thereby, the edge definition is close to, or smaller than the resolution limit of SRM, as can be seen by the red area of SRM images in Fig. 1B.

\section{Discussion of the interaction of the electron beam with the substrate and with oxo-G}

Fig. 3 shows that the electron beam interacts with the $\mathrm{SiO}_{2}$ substrate, as evident from the dark contrast of the uncoated area between the flakes. As discussed above, the correspondence of the BSE exit range with the observed range of reduction of the oxo-G suggests, that mainly low energy secondary electrons are responsible for the reduction of oxo-G to graphene.

The cause for the introduction of lattice defects in oxo-G/ graphene in close vicinity to the electron beam, on the other hand, is not so clear. Since oxo-G bears only a single layer carbon lattice, the direct interaction of the electron beam and oxo-G should be negligible. We therefore propose that the interaction of the electron beam with the $\mathrm{SiO}_{2}$ surface is determining the process. The resulting

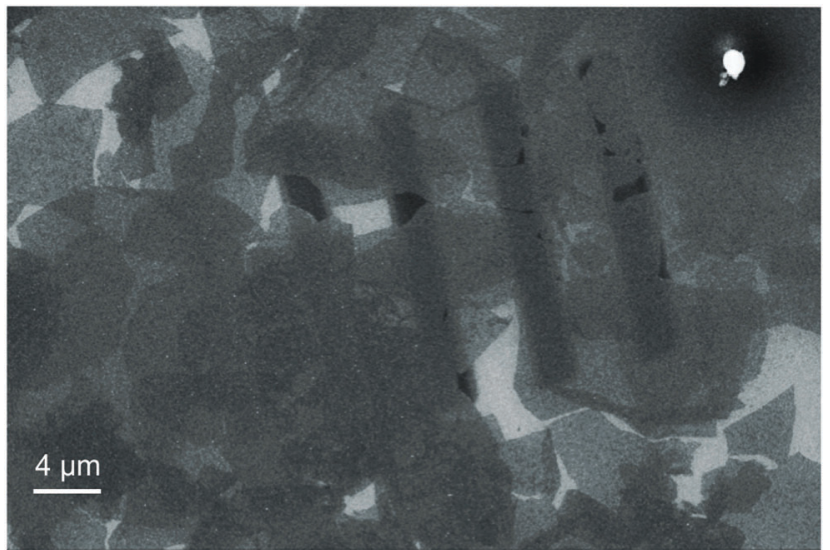

Fig. 3 Electron microscopy image recorded at $15 \mathrm{kV}$. Oxo-G flakes after irradiation (5 keV) and reduction. The electron irradiated striped areas are clearly visible as dark features. The dark features correspond to areas of reduced oxo-G by secondary electron emission and are mainly located on the bare $\mathrm{SiO}_{2}$. The observed contrast on the reduced oxo- $\mathrm{G}$ is likely due to modification of the underlying $\mathrm{SiO}_{2}$ as well.

low energy secondary electrons $(<50 \mathrm{eV} \text {, maximum } 5-8 \mathrm{eV})^{44,45}$ and higher energy electrons (BSEs, Auger electrons, low loss electrons) both could be responsible for dissociative processes at higher electron density, that is, in the immediate impingement area of the primary beam. The introduction of lattice defects (red color) is apparent for low energy primary beams (Fig. 1 and 2 at $5 \mathrm{kV}$ ); under these conditions a high density of low energy secondary electrons in the primary beam area is found in simulations. In addition, electron stimulated desorption of oxygen from $\mathrm{SiO}_{2}$ surfaces has been reported, ${ }^{36,46,47}$ and the oxidative decomposition of carbon frameworks is a common process. Thus, we propose that this release of reactive oxygen species from the $\mathrm{SiO}_{2}$ substrate could also induce $\mathrm{CO}$ or $\mathrm{CO}_{2}$ formation and therefore cause the degradation of the carbon lattice. Finally, defect formation could also be linked to beaminduced local heating, which could result in thermal decomposition of oxo-G and lattice rearrangement.

\section{Conclusions}

We find that electron beam treatment of oxo-G, and very likely also of conventional graphene oxide, leads to either reduction (reductive defunctionalization) to graphene, or to the introduction of in-plane lattice defects, depending on the irradiation conditions. Possible pathways for the defect formation leading to local oxo-G lattice degradation are reactions induced by slow secondary electrons at high electron densities, O-release from the $\mathrm{SiO}_{2}$ substrate, or local heating of the substrate. The formation of the defective carbon structure occurs most efficiently at low energy (best at $2 \mathrm{keV}$ and high dose of $800 \mathrm{mC} \mathrm{cm}^{-2}$ ) and occurs with high spatial confinement. On the other hand, at high energies, such as $30 \mathrm{keV}$, no local patterning of graphene is observed; instead, a reduction of large areas is detected, most likely due to the generation of slow secondary electrons that facilitate the reduction of oxo-G. Overall, we demonstrate that FEBIP is a suitable tool with high potential to pattern oxo-G on very small scales. 


\section{Acknowledgements}

The authors gratefully acknowledge financial support from the German Research Foundation (DFG) within the frameworks of the Collaborative Research Center SFB-953 Synthetic Carbon Allotropes, the Cluster of Excellence "Engineering of Advanced Materials", COST Action CM1301 (CELINA) and grant no. EI 938/3-1.

\section{References}

1 M. Garcia-Hernandez and J. Coleman, 2D Mater., 2016, 3, 010401.

2 K. S. Novoselov, V. I. Fal'ko, L. Colombo, P. R. Gellert, M. G. Schwab and K. Kim, Nature, 2012, 490, 192-200.

3 R. K. Joshi, S. Alwarappan, M. Yoshimura, V. Sahajwalla and Y. Nishina, Appl. Mater. Today, 2015, 1, 1-12.

4 G. Charpy, C. R. Hebd. Seances Acad. Sci., 1909, 148, 920-923.

5 H.-P. Boehm and W. Scholz, Liebigs Ann. Chem., 1966, 691, 1-8.

6 O. Jankovský, P. Marvan, M. Nováček, J. Luxa, V. Mazánek, K. Klímová, D. Sedmidubský and Z. Sofer, Appl. Mater. Today, 2016, 4, 45-53.

7 C. K. Chua and M. Pumera, Chem. Soc. Rev., 2014, 43, 291-312.

8 S. Eigler, C. Dotzer and A. Hirsch, Carbon, 2012, 50, 3666-3673.

9 R. Flyunt, W. Knolle, A. Kahnt, S. Eigler, A. Lotnyk, T. Häupl, A. Prager, D. Guldi and B. Abel, Am. J. Nano Res. Appl., 2014, 2, 9-18.

10 M. Baraket, S. G. Walton, Z. Wei, E. H. Lock, J. T. Robinson and P. Sheehan, Carbon, 2010, 48, 3382-3390.

11 T. Wu, S. Liu, H. Li, L. Wang and X. Sun, J. Nanosci. Nanotechnol., 2011, 11, 10078-10081.

12 S. Eigler, S. Grimm, M. Enzelberger-Heim, P. Müller and A. Hirsch, Chem. Commun., 2013, 49, 7391-7393.

13 A. M. Dimiev and S. Eigler, Graphene Oxide: Fundamentals and Applications, Wiley, 2016.

14 J. M. Englert, P. Vecera, K. C. Knirsch, R. A. Schafer, F. Hauke and A. Hirsch, ACS Nano, 2013, 7, 5472-5482.

15 S. Eigler, F. Hof, M. Enzelberger-Heim, S. Grimm, P. Müller and A. Hirsch, J. Phys. Chem. C, 2014, 118, 7698-7704.

16 M. M. Lucchese, F. Stavale, E. H. M. Ferreira, C. Vilani, M. V. O. Moutinho, R. B. Capaz, C. A. Achete and A. Jorio, Carbon, 2010, 48, 1592-1597.

17 L. G. Cançado, A. Jorio, E. H. M. Ferreira, F. Stavale, C. A. Achete, R. B. Capaz, M. V. O. Moutinho, A. Lombardo, T. S. Kulmala and A. C. Ferrari, Nano Lett., 2011, 11, 3190-3196.

18 S. Eigler, Chem. Commun., 2015, 51, 3162-3165.

19 S. Eigler, M. Enzelberger-Heim, S. Grimm, P. Hofmann, W. Kroener, A. Geworski, C. Dotzer, M. Rockert, J. Xiao, C. Papp, O. Lytken, H. P. Steinrück, P. Müller and A. Hirsch, Adv. Mater., 2013, 25, 3583-3587.

20 S. Eigler, Chem. - Eur. J., 2016, 22, 7012-7027.

21 B. Butz, C. Dolle, C. E. Halbig, E. Spiecker and S. Eigler, Angew. Chem., Int. Ed., 2016, 55, 15771-15774 (Angew. Chem., 2016, 128, 16003-16006).
22 J.-Y. Hong and J. Jang, J. Mater. Chem., 2012, 22, 8179-8191. 23 Y. Zhou, Q. Bao, B. Varghese, L. A. L. Tang, C. K. Tan, C.-H. Sow and K. P. Loh, Adv. Mater., 2010, 22, 67-71.

24 M. F. El-Kady, V. Strong, S. Dubin and R. B. Kaner, Science, 2012, 335, 1326-1330.

25 Z. Wei, D. Wang, S. Kim, S. Y. Kim, Y. Hu, M. K. Yakes, A. R. Laracuente, Z. Dai, S. R. Marder, C. Berger, W. P. King, W. A. de Heer, P. E. Sheehan and E. Riedo, Science, 2010, 328, 1373-1376.

26 Z. Sofer, P. Simek and M. Pumera, Phys. Chem. Chem. Phys., 2013, 15, 9257-9264.

27 O. Jankovský, Š. Hrdličková Kučková, M. Pumera, P. Šimek, D. Sedmidubský and Z. Sofer, New J. Chem., 2014, 38, 5700-5705.

28 S. Eigler, S. Grimm and A. Hirsch, Chem. - Eur. J., 2014, 20, 984-989.

29 S. H. Dave, C. Gong, A. W. Robertson, J. H. Warner and J. C. Grossman, ACS Nano, 2016, 10, 7515-7522.

30 T. Ishida, Y. Miyata, Y. Shinoda and Y. Kobayashi, Appl. Phys. Express, 2016, 9, 025103.

31 M. Cheng, R. Yang, L. Zhang, Z. Shi, W. Yang, D. Wang, G. Xie, D. Shi and G. Zhang, Carbon, 2012, 50, 2581-2587.

32 S. Grimm, M. Schweiger, S. Eigler and J. Zaumseil, J. Phys. Chem. C, 2016, 120, 3036-3041.

33 J. M. Mativetsky, E. Treossi, E. Orgiu, M. Melucci, G. P. Veronese, P. Samori and V. Palermo, J. Am. Chem. Soc., 2010, 132, 14130-14136.

34 I. Utke, P. Hoffmann and J. Melngailis, J. Vac. Sci. Technol., B: Microelectron. Nanometer Struct. - Process., Meas., Phenom., 2008, 26, 1197-1276.

35 W. F. van Dorp and C. W. Hagen, J. Appl. Phys., 2008, 104, 081301.

36 H. Marbach, Appl. Phys. A: Mater. Sci. Process., 2014, 117, 987-995.

37 C. E. Halbig, P. Rietsch and S. Eigler, Molecules, 2015, 20, 21050-21057.

38 R. Flyunt, W. Knolle, A. Kahnt, C. E. Halbig, A. Lotnyk, T. Haupl, A. Prager, S. Eigler and B. Abel, Nanoscale, 2016, 8, 7572-7579.

39 A. Kahnt, R. Flyunt, S. Naumov, W. Knolle, S. Eigler, R. Hermann and B. Abel, RSC Adv., 2016, 6, 68835-68845.

40 A. Kahnt, R. Flyunt, C. Laube, W. Knolle, S. Eigler, R. Hermann, S. Naumov and B. Abel, Nanoscale, 2015, 7, 19432-19437.

41 S. Eigler, Phys. Chem. Chem. Phys., 2014, 16, 19832-19835.

42 A. C. Ferrari, Solid State Commun., 2007, 143, 47-57.

43 D. Drouin, A. R. Couture, D. Joly, X. Tastet, V. Aimez and R. Gauvin, Scanning, 2007, 29, 92-101.

44 L. Reimer, in Scanning Electron Microsc., Springer-Verlag, Berlin - Heidelberg - New York, 2nd edn, 1998.

45 H. J. Fitting, J. Boyde and J. Reinhardt, Phys. Status Solidi A, 1984, 81, 323-332.

46 M. L. Knotek and P. J. Feibelman, Phys. Rev. Lett., 1978, 40, 964-967.

47 M. M. Walz, M. Schirmer, F. Vollnhals, T. Lukasczyk, H. P. Steinrück and H. Marbach, Angew. Chem., Int. Ed., 2010, 49, 4669-4673 (Angew. Chem., 2010, 122, 4774-4778). 